\title{
The Impact of a Value-Based Insurance Design Plus Health Coaching on Medication Adherence and Medical Spending
}

\author{
Shirley Musich, PhD, Sara Wang, PhD, and Kevin Hawkins, $\mathrm{PhD}^{1}$
}

\begin{abstract}
The objective of this study was to evaluate medication adherence, medical services utilization, and combined medical and pharmacy expenditures associated with diabetes and hypertension value-based insurance design (VBID) plus health/disease coaching programs implemented by a large employer. A pre/post participant versus nonparticipant study design was used to measure medication possession ratios (MPRs), inpatient admissions, emergency room utilization, and combined medical and pharmacy expenditures for employees/spouses with diabetes $(n=1090$; average 23 months follow-up) and hypertension $(n=3254$; average 13 months follow-up) participating in a VBID plus health/disease coaching relative to eligible nonparticipants. Outcome measures were propensity score weighted and regression adjusted to estimate the independent impact of the programs. MPRs for diabetes and hypertension were significantly increased 3 to 4 percentage points for VBID participants, while MPRs for respective nonparticipants decreased by about 10 percentage points. Employer-paid pharmacy expenditures increased significantly for both participants with diabetes and hypertension while outof-pocket patient co-payments decreased significantly. Medical expenditures for diabetes VBID participants decreased but not significantly. Hypertension participants experienced medical expenditure increases. Medical services utilization of inpatient admissions and emergency room visits underwent minimal change. Thus employer-sponsored diabetes and hypertension VBID plus health/disease coaching programs can be expected to lower patient co-payments and significantly increase medication adherence. Meanwhile, medical spending outcomes indicated that increased diabetes and hypertension pharmacy expenditures were partially offset by medical savings (for diabetes) but not sufficiently to be cost neutral. (Population Health Management 2015;18:151-158)
\end{abstract}

\section{Introduction}

$\mathbf{V}$ ALUE-BASED INSURANCE DESIGNS (VBIDs) have been used to improve quality of care, encouraging improved adherence to pharmaceutical protocols by selectively lowering patients' out-of-pocket medication spending. Generally, VBID programs provide reduced prescription drug co-payment costs for target chronic conditions such as diabetes, ${ }^{1-10}$ hypertension, ${ }^{1,6-10}$ hyperlipidemia, ${ }^{6,8-13}$ and asthma. ${ }^{1,7,10}$ VBID programs have been implemented successfully in both health plan $^{3,5,8,9,13}$ and employer markets. ${ }^{1,2,4,6,7,10-12}$

In recent years, consumer prescription drug price sharing and co-payment levels have increased steadily. The unintended consequence of these strategies was that as patient co-payments increased, medication adherence decreased. According to one meta-analysis, it was estimated that for every
$10 \%$ increase in cost sharing, prescription drug spending decreased by $2 \%$ to $6 \% .{ }^{14}$ In another study, every $\$ 10$ increase in patient cost sharing resulted in a $5.4 \%$ reduction in adherence to oral diabetic medications. ${ }^{15}$ Of clinical importance, it has been demonstrated that medication nonadherence was associated with documented adverse outcomes (eg, higher blood pressure, higher HbA1c levels among patients with diabetes, increased rates of diabetic complications, higher low-density lipoprotein cholesterol levels). ${ }^{15-17}$ Meanwhile, improved medication adherence was associated with fewer complications, lower medical spending, fewer emergency room visits, lower all-cause hospitalization rates, and lower all-cause mortality rates. ${ }^{14-16,18}$

Large employers have been experimenting with various VBID models for some time- the most notable of these being Pitney Bowes. Its VBID program was systematically designed

\footnotetext{
${ }^{1}$ Optum, Ann Arbor, Michigan.
} 
and subsequently evaluated to measure improved medication adherence for 2 selected drug classes (ie, cholesterollowering statins; clopidogrel, a blood clot inhibitor). ${ }^{10,11,19}$ Contrary to traditional insurance designs that price medications to the consumer based on their purchasing costs, VBID programs lower cost sharing for selected high-value medications with evidence-based outcomes. In a recent Mercer report, ${ }^{20}$ about $23 \%$ of large $(500+)$ employers indicated currently using VBID programs with $27 \%$ planning to use them in the future.

Although these plans are growing in popularity, currently no standard approach to VBID programs exists. There are variations in the chronic conditions targeted ${ }^{1-10}$ and, commonly, programs reduce selected generic drug co-payments to $\$ 0$ and reduce co-payments for other preferred/nonpreferred brand name drugs. ${ }^{5,6,8-10}$ However, other programs reduce coinsurance co-payment percentages based on established drug costs (eg, 10\% of prescription cost from $20 \%$ to $50 \%$ coinsurance rates $)^{1,2,7}$ or reduce co-payments to flat rates (eg, \$10 flat co-payment). ${ }^{3,4}$ More recently, some VBID programs have begun to include disease management coaching. ${ }^{2,6,10}$

A recent review of VBID programs ${ }^{21}$ concluded that VBID programs are generally successful in improving quality of care by increasing adherence to medication protocols. However, the expected program impact of reduced medical spending has been less well documented. The general consensus across several studies was that VBID programs were cost neutral, 1,2,6,12,21,22 generating sufficient medical savings to offset increased prescription drug spending. ${ }^{21}$

Because of employers' continued interest in the implementation of VBID models, the research team undertook the evaluation of a newly introduced diabetes and hypertension VBID program at a large employer. The purpose of the study was to evaluate the impact of the VBID program design plus health/disease coaching on medication adherence, medical services utilization, and medical and pharmacy expenditures. Key outcomes included comparisons of participants and nonparticipants with respect to: (1) medication possession ratios (MPRs), (2) utilization rates for inpatient admissions and emergency room visits, and (3) combined medical and pharmacy expenditures.

\section{Methods}

\section{Study design}

This study utilized a pre/post participant and nonparticipant comparison group design to evaluate a diabetes and hypertension VBID plus health/disease coaching program implemented by a large employer. The diabetes program initiated in 2010 utilized a baseline period 3 to 12 months prior to the enrollment date with 3 to 36 months (average 23 months) of follow-up through June 2013. The hypertension program initiated in 2011 utilized similar baseline criteria prior to the enrollment date and 3 to 24 months (average 13 months) of follow-up through June 2013.

Participation in the program was documented with enrollment dates and from payments made by the pharmaceutical benefit management (PBM) provider. Eligible nonparticipants must have used at least 1 prescription within the respective therapeutic classes or had at least 2 diagnosis codes to confirm the target diagnosis. The difference- in-difference (DID) design compared pre to post defined medication adherence and medical spending outcomes for VBID participants and nonparticipants.

\section{Sample}

Employees and spouses enrolled in lifestyle management health coaching or disease management coaching programs who had been diagnosed with either diabetes or hypertension were eligible for the VBID programs. The lifestyle management and disease management programs were part of a broader corporate health management program. Eligible VBID participants and nonparticipants must have had at least 3 months of continuous medical plan enrollment prior to their enrollment date and at least 3 months of continuous enrollment after enrollment (ie, a minimum of 6 months of continuous medical enrollment).

To better understand the costs associated with patients who regularly use pharmaceuticals to manage their condition (ie, chronic pharmacy utilizers), the research team implemented an additional criterion that VBID participants and nonparticipants must have had at least 2 prescriptions in the pre and post time periods within the respective therapeutic classes. Outliers were removed to equalize the total health care and pharmacy expenditure distributions between participants and nonparticipants in the pre and post periods, resulting in the exclusion of $3 \%$ of observations. Maternity cases also were excluded. The final study populations included 814 diabetes VBID employee/spouse participants and 276 diabetes nonparticipant controls and 2674 hypertension VBID participants and 580 hypertension nonparticipant controls. Study groups were not mutually exclusive, thus allowing individuals to be enrolled in either or both of the chronic condition programs.

\section{VBID plus health/disease coaching program}

Eligible VBID participants and nonparticipants must have been currently enrolled in either lifestyle management or disease management coaching programs and referred to the VBID program by the respective health/disease coaches. Coverage for diabetic and hypertension medications and diabetic supplies were made available with reduced or eliminated co-payments. Similar to other plans, generic drug copayments were eliminated (ie, \$0). Preferred brands were available with $\$ 5$ co-payments for a 34-day supply or $\$ 15$ for a 90-day supply. Non-preferred brands were available at $50 \%$ coinsurance rates with applicable minimum/maximum levels. Diabetic supplies were made available at no cost to members with diabetes.

\section{Measures}

Outcome measures. Key outcome measures for this evaluation included pre/post comparisons of: (1) MPRs, (2) medical services utilization (ie, inpatient admissions, emergency room visits), and (3) combined medical and pharmacy expenditures for participants and nonparticipants, respectively.

Medication possession ratios. Medication adherence was defined as MPRs based on the percentage of days that a member had his or her medication available over the pre 
period and the subsequent time enrolled in the program. The dates when prescriptions were filled and the number of days' supply on the prescription drug claims were used to determine how many days medications were on hand. The research team calculated the ratio as a combined metric for all drugs prescribed within a therapeutic class. Post period results are presented as 2-year averages for participants with diabetes and as a 1-year average for those with hypertension. Use of electronic pharmacy records to determine medication adherence and/or nonadherence have been previously validated comparing patient reports, pharmacy records, and pill counts to verify pharmacy records. ${ }^{23}$

Medical services utilization. All-cause medical services utilization rates for inpatient admissions and emergency room visits were calculated from place of service codes within medical claims. Emergency room visits and inpatient admission utilization rates were calculated for the pre period prior to enrollment and for the follow-up post periods for participants and nonparticipants with diabetes and hypertension, respectively. Because baseline and follow-up periods could be variable, rates were annualized for participant and nonparticipant comparisons (ie, total inpatient or emergency room events divided by the total member years).

Health care and pharmacy costs. Health care expenditures (per member per month [PMPM]) were calculated in US dollars for each eligible employee and spouse, including all inpatient, outpatient, professional, and pharmacy paid claims, for at least 3 months and up to 12 months prior to the enrollment date and for at least 3 months and up to 36 months (diabetes) or 24 months (hypertension) after the enrollment date. All costs were adjusted to 2013 dollars using the medical care services component in the Consumer Price Index. Employer-paid claims were used to provide medical and pharmacy expenditure trend outcomes, enabling the employer to assess potential medical savings relative to the investment in additional pharmacy coverage. Pharmaceutical co-payments for participants and nonparticipants were calculated separately to document changes in patient copayments after enrollment in the VBID program.

Covariates. Covariates were included to adjust for other factors that may influence the selection bias often associated with program participation. These covariates included measures of demographics, health status, and other characteristics taken from health plan eligibility and claims files. Demographic variables included the participant's age, sex, and location. Age was stratified into 4 groups (age: 18-34, 35-44, 45-54, and 55+ years). Insurance plans included: Blue Cross Blue Shield, Aetna, UnitedHealthcare, and Other. Income and location were geocoded from zip codes to: High, Upper Medium, Lower Medium, and Low for income; Metropolitan and Other for location. The number of available months in the pre and post periods were added to account for different lengths of time in the programs.

Health status covariates were measured from claims data and included the calculated Charlson Comorbidity Index $(\mathrm{CCI}),{ }^{24}$ Psychiatric Diagnostic Group score (PDG),${ }^{25}$ and the annual number of physician office visits, emergency room visits, and inpatient admissions. The $\mathrm{CCI}$ is a measure of the risk of 1-year all-cause mortality attributable to se- lected comorbidities that also has been shown to be highly predictive of morbidity and health care expenditures. The PDG score includes validated psychiatric diagnostic groups analogous to major diagnostic groups in the diagnosticsrelated group system but provides better classification of individuals with substance abuse and/or mental health disorders. Measures of health services availability included: acute hospital beds per 1000 and primary care physicians per 100,000. Differences in covariates between participants and nonparticipants were tested with chi-square tests for categorical variables or Student $t$ tests for continuous variables.

\section{Statistical analyses}

Propensity score weighting. Propensity score weighting used information about the demographic, socioeconomic, and health status variables already described to adjust for potential selection bias often associated with participation in programs, thereby allowing the comparison of participants to similar nonparticipants. This information was used to estimate the underlying probability of VBID program participation for each individual. The research team then used that estimated probability to create a weighting variable applied to the data from those who chose not to participate in the programs, to make them better resemble all eligible employees/spouses. The value of the weighting variable equals 1 /predicted probability of specific program participation. The utility of propensity score models to adjust for external validity threats are described elsewhere. ${ }^{26,27}$

Regression modeling. After propensity score weighting, statistical differences between diabetes and hypertension VBID program participants and nonparticipants are expected to be minimized or eliminated. Any remaining differences, however, were subsequently adjusted for using generalized linear regression models as a final adjustment in comparing the outcome variables as reported elsewhere. ${ }^{28}$ Differences in the weighted outcome measures (ie, medical expenditures, MPRs, utilization measures) between participants and nonparticipants (ie, DID) thus controlled for demographics, health status, and health plan characteristics.

\section{Results}

Take-up rates for the VBID programs were very high among those chronic pharmacy utilizers identified for the evaluation: $75 \%$ of those with diabetes and $82 \%$ of those with hypertension. Baseline characteristics of participants and nonparticipants in the diabetes and hypertension VBID programs are shown in Tables 1 and 2. Participants in the diabetes and hypertension programs were less healthy than their respective nonparticipant controls (eg, higher CCI scores, higher medical utilization), further documenting the need for propensity score weighting. The weighting worked well, as the significant differences between participants and respective nonparticipant controls were generally removed or minimized as illustrated in Table 1 and Table 2, respectively. Because some case-mix differences still remained between the groups, subsequent regression adjustments were warranted to further minimize these differences.

Comparisons of descriptive unadjusted MPRs for participants with diabetes indicated that medication adherence 
Table 1. Baseline Characteristics of Diabetes VBID Participants and Nonparticipants Before and After Propensity Score Weighting

\begin{tabular}{|c|c|c|c|c|c|c|}
\hline & \multicolumn{3}{|c|}{ Unweighted } & \multicolumn{3}{|c|}{ Propensity Score Weighted } \\
\hline & \multirow{2}{*}{$\frac{\text { Participant }}{\text { Mean or } \%}$} & \multicolumn{2}{|c|}{ Nonparticipant } & \multirow{2}{*}{$\frac{\text { Participant }}{\text { Mean or } \%}$} & \multicolumn{2}{|c|}{ Nonparticipant } \\
\hline & & Mean or \% & $\mathrm{P}$ value & & Mean or $\%$ & $\mathrm{P}$ value \\
\hline & 814 & 276 & & 814 & 276 & \\
\hline Age (average age) & 49.6 & 47.4 & 0.001 & 49.0 & 49.3 & 0.69 \\
\hline $18-34$ & 6.5 & 7.3 & 0.001 & 6.5 & 6.1 & 0.99 \\
\hline $35-44$ & 23.1 & 33.7 & & 25.5 & 25.2 & \\
\hline $45-54$ & 36.0 & 36.2 & & 36.3 & 37.2 & \\
\hline $55+$ & 34.4 & 22.8 & & 31.7 & 31.6 & \\
\hline \multicolumn{7}{|l|}{ Sex } \\
\hline Female & 36.1 & 36.6 & 0.88 & 35.8 & 33.8 & 0.55 \\
\hline Male & 63.9 & 63.4 & & 64.2 & 66.2 & \\
\hline \multicolumn{7}{|l|}{ Relationship } \\
\hline Employee & 80.8 & 80.8 & 0.99 & 80.6 & 84.1 & 0.20 \\
\hline \multirow{2}{*}{\multicolumn{7}{|c|}{ Health plan }} \\
\hline & & & & & & \\
\hline Aetna & 41.2 & 45.3 & 0.0001 & 42.5 & 42.3 & 0.19 \\
\hline BCBS & 31.6 & 22.1 & & 30.0 & 34.5 & \\
\hline UHC & 23.7 & 23.6 & & 23.2 & 17.7 & \\
\hline Other & 3.6 & 9.1 & & 4.4 & 5.6 & \\
\hline \multicolumn{7}{|l|}{ Income } \\
\hline High & 66.2 & 64.5 & 0.41 & 65.4 & 65.3 & 0.16 \\
\hline Upper Medium & 15.7 & 15.6 & & 16.0 & 15.6 & \\
\hline Lower Medium & 7.1 & 10.5 & & 7.2 & 10.9 & \\
\hline Low & 3.8 & 4.0 & & 4.0 & 3.8 & \\
\hline \multicolumn{7}{|l|}{ Location } \\
\hline Metropolitan & 95.0 & 94.6 & 0.80 & 95.1 & 94.1 & 0.53 \\
\hline Other & 5.0 & 5.4 & & 4.9 & 5.9 & \\
\hline Inpatient admissions (annual \%) & 6.8 & 3.3 & 0.01 & 5.9 & 7.1 & 0.48 \\
\hline Emergency room visits (annual \%) & 17.8 & 11.2 & 0.005 & 16.7 & 13.6 & 0.24 \\
\hline Physician office visits per year & 6.5 & 5.9 & 0.07 & 6.3 & 6.3 & 0.91 \\
\hline Acute hospital beds per 1000 & 2.0 & 2.0 & 0.94 & 2.0 & 2.0 & 0.96 \\
\hline Primary care physicians per 100,000 & 67.2 & 67.3 & 0.93 & 67.0 & 68.3 & 0.27 \\
\hline Pre period (months) & 11.2 & 10.7 & 0.007 & 11.0 & 10.9 & 0.75 \\
\hline Post period (months) & 22.6 & 28.9 & $<0.0001$ & 22.6 & 27.6 & $<0.0001$ \\
\hline Charlson Comorbidity Index (CCI) & 1.5 & 1.2 & $<0.0001$ & 1.4 & 1.4 & 0.36 \\
\hline $\mathrm{CCI}=0$ & 4.7 & 17.8 & $<0.0001$ & 7.9 & 7.7 & 0.90 \\
\hline $\mathrm{CCI}=1$ & 62.9 & 58.3 & 0.18 & 61.7 & 60.5 & 0.71 \\
\hline CCI $\geq 2$ & 32.4 & 23.9 & 0.008 & 30.3 & 31.9 & 0.64 \\
\hline Psychiatric Diagnostic Group & 0.2 & 0.2 & 0.27 & 0.2 & 0.2 & 0.65 \\
\hline No $($ score $=0)$ & 84.9 & 81.9 & 0.24 & 84.3 & 85.7 & 0.56 \\
\hline Yes $($ score $\geq 1)$ & 15.1 & 18.1 & 0.24 & 15.7 & 14.3 & 0.56 \\
\hline
\end{tabular}

BCBS, Blue Cross Blue Shield; UHC, UnitedHealthcare; VBID, value-based insurance design

improved by 4 percentage points for participants while medication adherence for nonparticipants decreased by about 10 percentage points, as illustrated in Figure 1. Similarly, MPRs for participants with hypertension increased by about 3 percentage points and decreased for nonparticipants by about 9 percentage points. Regression-adjusted weighted DIDs for MPRs comparing participant and nonparticipant trends indicated a significant 14.1 percentage point gain for participants with diabetes $(P<0.0001)$ and a significant 14.3 percentage point gain for participants with hypertension $(P<0.0001)$ as illustrated in Tables 3 and 4 , respectively.

Adjusted health care expenditures for participants with diabetes indicated significantly increased pharmacy costs relative to nonparticipants $(P<0.0001)$ and small but nonsignificant savings in medical costs $(P=0.58)$, as shown in Table 3. Overall, although savings in medical expenditures for participants with diabetes partially offset increased pharmaceutical payments, those savings were not sufficient for the program to be considered cost neutral. However, adjusted combined medical and pharmacy expenditure DIDs comparing participants and nonparticipants were not statistically significant $(P=0.48)$. Participants with diabetes, meanwhile, experienced significant co-payment reductions: $\$ 20.60$ savings per month or about $\$ 250$ per year $(P<0.0001$; data not shown). Annualized adjusted differences for inpatient admissions and emergency room utilization rates for participants with diabetes indicated no 
TAble 2. Baseline Characteristics of Hypertension VBID Participants and Nonparticipants Before and After Propensity Score Weighting

\begin{tabular}{|c|c|c|c|c|c|c|}
\hline \multirow[b]{4}{*}{ Hypertension } & \multicolumn{3}{|c|}{ Unweighted } & \multicolumn{3}{|c|}{ Propensity Score Weighted } \\
\hline & \multirow{2}{*}{$\frac{\text { Participant }}{\text { Mean or } \%}$} & \multicolumn{2}{|c|}{ Nonparticipant } & \multirow{2}{*}{$\frac{\text { Participant }}{\text { Mean or } \%}$} & \multicolumn{2}{|c|}{ Nonparticipant } \\
\hline & & Mean or \% & $\mathrm{P}$ value & & Mean or \% & $\mathrm{P}$ value \\
\hline & 2674 & 580 & & 2674 & 580 & \\
\hline Age (average age) & 49.8 & 49.3 & 0.20 & 49.8 & 50.1 & 0.46 \\
\hline $18-34$ & 5.2 & 4.8 & 0.41 & 5.2 & 5.6 & 0.44 \\
\hline $35-44$ & 24.1 & 24.8 & & 23.8 & 20.6 & \\
\hline $45-54$ & 38.0 & 40.9 & & 38.5 & 39.9 & \\
\hline $55+$ & 32.8 & 29.5 & & 32.5 & 34.0 & \\
\hline \multicolumn{7}{|l|}{ Sex } \\
\hline Female & 31.4 & & $<0.0001$ & 33.5 & 34.3 & 0.68 \\
\hline Male & 68.6 & 57.2 & & 66.6 & 65.7 & \\
\hline \multicolumn{7}{|l|}{ Relationship } \\
\hline Employee & 87.2 & 65.5 & $<0.0001$ & 82.6 & 81.2 & 0.40 \\
\hline Spouse & 12.8 & 34.5 & & 17.4 & 18.8 & \\
\hline \multicolumn{7}{|l|}{ Health plan } \\
\hline BCBS & 29.9 & 28.5 & 0.72 & 29.8 & 27.2 & 0.03 \\
\hline UHC & 58.2 & 58.8 & & 57.4 & 56.0 & \\
\hline Other & 11.9 & 12.8 & & 12.8 & 16.8 & \\
\hline \multicolumn{7}{|l|}{ Income } \\
\hline High & 71.4 & 74.7 & 0.47 & 72.1 & 72.4 & 0.63 \\
\hline Upper Medium & 12.8 & 12.1 & & 12.6 & 14.1 & \\
\hline Lower Medium & 6.9 & 5.2 & & 6.6 & 6.3 & \\
\hline Low & 2.8 & 2.4 & & 2.7 & 2.6 & \\
\hline Missing & 6.1 & 5.7 & & 6.1 & 4.7 & \\
\hline \multicolumn{7}{|l|}{ Location } \\
\hline Metropolitan & 95.6 & 96.2 & 0.48 & 95.6 & 95.6 & 0.94 \\
\hline Other & 4.5 & 3.8 & & 4.4 & 4.5 & \\
\hline Inpatient admissions (annual \%) & 5.0 & 4.8 & 0.88 & 5.3 & 6.9 & 0.15 \\
\hline Emergency room visits (annual \%) & 15.6 & 15.3 & 0.86 & 15.8 & 18.5 & 0.11 \\
\hline Physician office visits per year & 5.7 & 5.6 & 0.55 & 5.7 & 6.0 & 0.32 \\
\hline Acute care hospital beds per 1000 & 2.0 & 1.9 & 0.002 & 2.0 & 2.0 & 0.50 \\
\hline Primary care physicians per 100,000 & 68.2 & 69.8 & 0.08 & 68.4 & 68.9 & 0.56 \\
\hline Pre period (months) & 11.5 & 11.2 & 0.003 & 11.4 & 11.5 & 0.76 \\
\hline Post period (months) & 13.3 & 18.2 & $<0.0001$ & 13.3 & 19.1 & $<0.0001$ \\
\hline Charlson Comorbidity Index (CCI) & 0.67 & 0.45 & $<0.0001$ & 0.65 & 0.73 & 0.08 \\
\hline $\mathrm{CCI}=0$ & 58.7 & 69.7 & $<0.0001$ & 59.9 & 55.4 & 0.04 \\
\hline $\mathrm{CCI}=1$ & 25.5 & 21.0 & 0.02 & 25.1 & 25.7 & 0.79 \\
\hline $\mathrm{CCI} \geq 2$ & 15.8 & 9.3 & $<0.0001$ & 15.0 & 19.0 & 0.02 \\
\hline Psychiatric Diagnostic Group & 0.25 & 0.35 & 0.001 & 0.26 & 0.30 & 0.14 \\
\hline No $($ score $=0)$ & 80.3 & 74.0 & 0.001 & 79.2 & 77.3 & 0.31 \\
\hline Yes (score $\geq 1$ ) & 19.8 & 26.0 & 0.001 & 20.8 & 22.7 & 0.31 \\
\hline
\end{tabular}

BCBS, Blue Cross Blue Shield; UHC, UnitedHealthcare; VBID, value-based insurance design

significant differences over the follow-up period compared to nonparticipants.

As with participants with diabetes, employer-paid pharmacy expenditures significantly increased for participants with hypertension $(P<0.0001)$ but, with the shorter followup time period, medical expenditures also increased, although not significantly $(P=0.34)$, as shown in Table 4 . Overall, the adjusted DID for combined medical and pharmacy expenditures for participants and nonparticipants with hypertension were not statistically significant $(P=0.11)$. Pharmaceutical co-payments for participants with hypertension decreased significantly by $\$ 8.90$ per month or about $\$ 110$ per year $(P \leq 0.0001$; data not shown). Annualized adjusted differences for inpatient admissions and emergency room utilization rates for participants with hypertension indicated significant increases in both inpatient and emergency room use over the follow-up period compared to nonparticipants.

\section{Discussion}

The VBID plus health/disease coaching program design implemented by this large employer leveraged information suggested by the scientific literature that VBID programs are more effective if program designs include coaching options. $^{2,6,10}$ The present evaluation included several methodological improvements to more reliably evaluate medical and pharmacy utilization and expenditure outcomes. Eligible nonparticipants within the same company were used as controls for this study design. This assured equivalent 


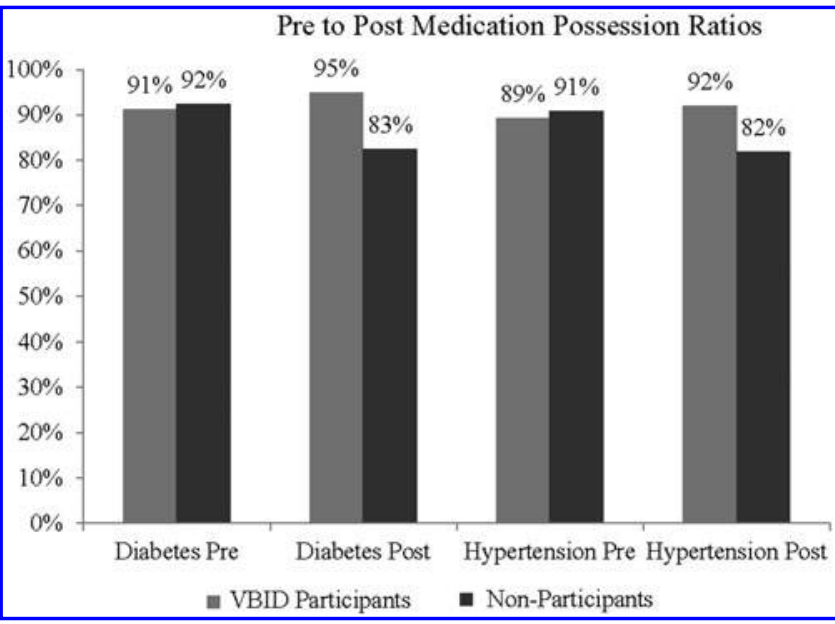

FIG. 1. Unadjusted descriptive pre to post MPRs for diabetes and hypertension VBID participants and nonparticipants.

Diabetes: $\mathrm{N}=814$ participants/276 nonparticipants.

Hypertension: $\mathrm{N}=2674$ participants/580 nonparticipants. MPR, medication possession ratio; VBID, value-based insurance design

corporate benefits, health messaging, and optional health management programs across both participants and controls. The take-up rates for the VBID programs were very high, a tribute to the recruitment and referral strategies of the health/disease coaches and to the perceived value of the VBID co-payment reduction design. Some VBID designs minimize pharmacy co-payment reductions with lowered coinsurance rates rather than elimination of some co-payments (especially for high-value generic medications). ${ }^{2,7}$ Rather than population-based evaluations, the research team documented VBID participation with documented enrollment dates and payment verification by the PBM provider. Use of propensity score weighting and subsequent regression adjustments, and exclusion of outliers and maternity cases improved on previous methodologies utilized in the measurement of health care expenditure trends over time. ${ }^{21}$

Key outcomes for VBID programs generally focus on changes in patient pharmacy co-payments (substantially decreased) and significantly improved medication adherence. MPRs for both participants with diabetes and hypertension increased about 3 to 4 percentage points-in the general range of what is reported in the literature for other VBID studies. ${ }^{2,4-6,8,9}$ Some VBID programs have indicated that time is required to build on medication adherence rates or that medication adherence improvements may not be sustainable. ${ }^{1,2,9}$ The present program showed relatively consistent MPRs over the respective follow-up periods, likely a benefit of the coaching interactions integrated into the program.

However, nonparticipants in both programs, decreased medication adherence by about 10 percentage points. This increase in nonadherence over time among nonparticipants has been noted by other researchers. ${ }^{5,6,8,9}$ Thus, the medication adherence advantages of VBID participants relative to nonparticipants apparently includes not only increased medication adherence with decreased pharmacy co-payments but also functions to prevent pharmacy discontinuation (ie, increasing rates of nonadherence over time). ${ }^{5}$ The overall gain in medication adherence for participants with diabetes or hypertension, relative to controls, was about 14 percentage points.

Medical services utilization including both inpatient admissions and emergency room visits was minimally impacted. It is possible that longer term studies are needed in order to demonstrate a program impact of reduced emergency room visits or inpatient admissions among employee/ spouse populations. In this evaluation, participants with diabetes with an average of about 2 years of follow-up showed changes in utilization that were not statistically significant after adjustments for covariates. In contrast, the hypertension program with about 1 year of follow-up showed a short-term increase in medical utilization likely because of increased focus on management of the condition. Perhaps longer (eg, 3-5 year) follow-ups may be needed - although, to date, only $1-$, $^{3,6,8,10,11,13} 2-,{ }^{4,9}$ or 3 -year ${ }^{1,2,7}$ VBID studies have been published.

VBID programs are used to improve quality of care by encouraging improved adherence to prescription drug medications. Although such programs are often evaluated for cost efficiency, profitability is not necessarily a requirement for success. For example, several VBID studies

Table 3. Differences in MPRs, Medical Utilization Rates, and Combined Medical and Pharmacy ExPENDitures for Diabetes VBID Participants Relative to NonParticipants

\begin{tabular}{|c|c|c|c|c|c|c|c|c|}
\hline & \multicolumn{8}{|c|}{ Diabetes VBID } \\
\hline & \multicolumn{2}{|c|}{$\begin{array}{l}\text { Combined Medical } \\
\text { Expenditures (Paid) }\end{array}$} & \multicolumn{2}{|c|}{$M P R s$} & \multicolumn{2}{|c|}{$\begin{array}{c}\text { Inpatient } \\
\text { Admissions }\end{array}$} & \multicolumn{2}{|c|}{$\begin{array}{l}\text { Emergency } \\
\text { Room Visits }\end{array}$} \\
\hline & $D I D(P M P M)$ & $\mathrm{P}$ value & $D I D^{*}$ & $\mathrm{P}$ value & $D I D^{*}$ & $\mathrm{P}$ value & $D I D^{*}$ & $\mathrm{P}$ value \\
\hline Descriptive Unadjusted & $-\$ 17.30$ & 0.74 & 13.6 & $<0.0001$ & -2.7 & 0.10 & -6.7 & 0.01 \\
\hline Descriptive Weighted by PS & $-\$ 91.30$ & 0.08 & 13.4 & $<0.0001$ & 1.9 & 0.34 & -2.5 & 0.40 \\
\hline Medical Expenditures & $\$ 29.00$ & 0.58 & & & & & & \\
\hline Pharmacy Expenditures & $-\$ 120.30$ & $<0.0001$ & & & & & & \\
\hline Regression Adjusted Weighted by PS & $-\$ 75.13$ & 0.48 & 14.1 & $<0.0001$ & 2.4 & 0.24 & -1.0 & 0.74 \\
\hline
\end{tabular}

$\mathrm{N}=814$ participants and 276 nonparticipants.

*DID percentage point difference in MPRs or annualized medical services utilization rates.

DID, difference in difference; MPR, medication possession ratio; PMPM, per member per month; PS, propensity score; VBID, valuebased insurance design 
Table 4. Differences in MPRs, Medical Utilization Rates, and Combined Medical and Pharmacy Expenditures for Hypertension VBID Participants Relative to NonParticipants

\begin{tabular}{|c|c|c|c|c|c|c|c|c|}
\hline & \multicolumn{8}{|c|}{ Hypertension VBID } \\
\hline & \multicolumn{2}{|c|}{$\begin{array}{l}\text { Combined Medical } \\
\text { Expenditures (Paid) }\end{array}$} & \multicolumn{2}{|c|}{ MPRs } & \multicolumn{2}{|c|}{$\begin{array}{l}\text { Inpatient } \\
\text { Admissions }\end{array}$} & \multicolumn{2}{|c|}{$\begin{array}{l}\text { Emergency } \\
\text { Room Visits }\end{array}$} \\
\hline & $D I D(P M P M)$ & $\mathrm{P}$ value & $D I D^{*}$ & $\mathrm{P}$ value & $D I D^{*}$ & $\mathrm{P}$ value & $D I D^{*}$ & $\mathrm{P}$ value \\
\hline Descriptive Unadjusted & $-\$ 74.98$ & 0.02 & 11.5 & $<0.0001$ & 1.0 & 0.40 & 1.5 & 0.43 \\
\hline Descriptive Weighted by PS & $-\$ 70.08$ & 0.03 & 13.7 & $<0.0001$ & 1.8 & 0.16 & 2.8 & 0.17 \\
\hline Medical Expenditures & $-\$ 30.41$ & 0.34 & & & & & & \\
\hline Pharmacy Expenditures & $-\$ 39.72$ & $<0.0001$ & & & & & & \\
\hline Regression Adjusted Weighted by PS & $-\$ 75.91$ & 0.11 & 14.3 & $<0.0001$ & 3.5 & 0.02 & 5.0 & 0.04 \\
\hline
\end{tabular}

$\mathrm{N}=2674$ participants and 580 nonparticipants.

*DID percentage point differences in MPRs or annualized medical services utilization rates.

DID, difference in difference; MPR, medication possession ratio; PMPM, per member per month; PS, propensity score; VBID, valuebased insurance design

considered successful have concluded that their programs were cost neutral. ${ }^{1,2,6,10,12,21}$ Although the diabetes program did show some medical cost savings (about $\$ 350$ per member per year), that was not enough savings to offset the increased pharmacy expenditures by the employer (about $\$ 1445$ per member per year). The average age of participants was about 50 years in a relatively highly educated workforce with multiple options for other health management programs. In this environment, it may take more time for the investment in additional medication consumption to provide a documented return on that investment. In their recent review of published VBID studies Lee et $\mathrm{al}^{21}$ concluded that, given the various program designs, study time lines, and analytic methodologies, the programs do not increase or decrease medical cost trends. The present study would fall within those parameters in that combined medical and pharmacy expenditures changes (ie, increases) associated with the programs were not statistically significant.

\section{Limitations}

Propensity score weighting and regression adjustments were utilized to account for differences between participant and nonparticipant populations in order to evaluate the impact of the VBID program on health care utilization and expenditures for pharmaceuticals and health care spending. However, additional unmeasured differences may still exist between the populations (eg, attitudes toward health, engagement levels). The research team did not have information on the disease stages but, with an average age of about 50, most individuals likely would be in the early stages of their disease. This justifies the program focus on prevention and improved management but will necessitate longer term studies with additional years of follow-up to demonstrate potential positive cost outcomes. 1,2,6,12,21,22 Using nonparticipants from the same company who were eligible but chose not to enroll in the VBID program ensured that these individuals had the same benefits programs, similar health messaging, and the advantages of the same on-site and online health management programs. Furthermore, the research team qualified the study population (participants and nonparticipants) as chronic pharmacy utilizers by requiring at a minimum at least 2 prescriptions pre and post to further ensure equivalent comparison populations.

This VBID program design included health/disease coaching and was integrated into a well-designed, multiyear health management program targeting a younger, healthier workforce. The combination of the VBID program with coaching provided a highly effective initial take-up of the program with immediate medication adherence improvements. However, these results may not generalize to other employer groups with different workforce characteristics and/or limited options for health management programming.

\section{Conclusions}

This newly implemented VBID program significantly reduced pharmacy co-payments for participants and significantly increased medication adherence for both participants with diabetes and hypertension while nonparticipants had a significant medication adherence drop-off. Inpatient admissions and emergency room utilization were not significantly impacted. Significantly increased pharmacy expenditures (both diabetes and hypertension medications) were partially offset by medical savings (for diabetes) but not sufficiently to be considered cost neutral.

\section{Author Disclosure Statement}

Drs. Musich, Wang, and Hawkins declared no conflicts of interest with respect to the research, authorship, and/or publication of this article. The authors received the following financial support for the research, authorship, and/or publication of this article: The source of funding for the study was a large technology employer.

\section{References}

1. Gibson TB, Wang S, Kelly E, et al. A value-based insurance design program at a large company boosted medication adherence for employees with chronic disease. Health Aff (Millwood). 2011;30:109-117.

2. Gibson TB, Mahoney J, Ranghell K, Cherney BJ, McElwee N. Value-based insurance plus disease management increased medication use and produced savings. Health Aff (Millwood). 2011;30:100-108. 
3. Zeng F, An JJ, Scully R, Barrington C, Patel BV, Nichol MB. The impact of value-based benefit design on adherence to diabetes medications: a propensity score-weighted difference in difference evaluation. Value Health. 2010;13: 846-852.

4. Nair KV, Miller K, Park J, Allen RR, Saseen JJ, Biddle V. Prescription co-pay reduction program for diabetic employees. Popul Health Manag. 2010;13:235-245.

5. Chang A, Leberman JN, Coulen C, Berger JE, Brennan TA. Value-based insurance design and antidiabetic medication adherence. Am J Pharm Benefits. 2010;2:39-44.

6. Kim YA, Loucks A, Yokoyama G, Lightwood J, Rascate K, Serxner SA. Evaluation of value-based insurance design with a large retail employer. Am J Manag Care. 2011;17: 682-690.

7. Kelly EJ, Turner CD, Frech-Tamas FH, Doyle JJ, Mauceri EG. Value-based benefit design and healthcare utilization in asthma, hypertension, and diabetes. Am J Pharm Benefits. 2009;1:217-221.

8. Maciejewski ML, Farley JF, Parker J, Wansink D. Copayment reductions generate greater medication adherence in targeted patients. Health Aff (Millwood). 2010;29:20022008.

9. Farley JF, Wansink D, Lindquist JH, Parker JC, Maciejewski ML. Medication adherence changes following valuebased insurance design. Am J Manag Care. 2012;18:265274.

10. Chernew ME, Shah MR, Wegh A, et al. Impact of decreasing copayments on medication adherence within a disease management environment. Health Aff (Millwood). 2008;27:103-112.

11. Choudhry NK, Fischer MA, Avorn J, et al. At Pitney Bowes, value-based insurance design cut copayments and increased drug adherence. Health Aff (Millwood). 2010;29: 1995-2001.

12. Choudhry NK, Fischer MA, Avorn JL, et al. The impact of reducing cardiovascular medication copayments on health spending and resource utilization. J Am Coll Cardiol. 2012; 60:1817-1824.

13. Frank MB, Fendrick AM, He Y, et al. The effect of a large regional health plan's value-based insurance design program on statin use. Med Care. 2012;50:934-939.

14. Goldman DP, Joyce GF, Zheng Y. Prescription drug cost sharing: associations with medication utilization and spending and health. JAMA. 2007;298:61-69.

15. Gibson TB, Song X, Alemayehu B, et al. Cost sharing, adherence, and health outcomes in patients with diabetes. Am J Manag Care. 2010;16:589-600.

16. Ho PM, Rumsfeld JS, Masoudi FA, et al. Effect of medication nonadherence on hospitalization and mortality among patients with diabetes mellitus. Arch Intern Med. 2006;166: 1836-1841.

17. Hunt J, Rozenfeld Y, Shenolikar R. Effect of patient medication cost share on adherence and glycemic control. Manag Care. 2009;18:47-53.

18. Sokol MC, McGuigan KA, Vergrugge RR, Epstein RS. Impact of medication adherence on hospitalization risk and healthcare cost. Med Care. 2005;43:521-30.

19. Mahoney JJ. Value-based benefit design: using a predictive modeling approach to improve compliance. J Manag Care Pharm. 2008;14(6 suppl B):S3-S8.

20. Mercer. Mercer's National Survey of Employer-Sponsored Health Plans. February 2013. http://benefitcommunications .com/upload/downloads/Mercer_Survey_2013.pdf. Accessed January 18, 2014.

21. Lee JL, Macijewski ML, Raju SS, Shrank WH, Choudhry NK. Value-based insurance design: quality improvement but no cost savings. Health Aff (Millwood). 2013;32:12511257.

22. Chernew ME, Juster IA, Shah M, et al. Evidence that valuebased insurance can be effective. Health Aff (Millwood). 2010;29:530-536.

23. Choo PW, Rand CS, Inui TS, et al. Validation of patient reports, automated pharmacy records, and pill counts with electronic monitoring of adherence to antihypertensive therapy. Med Care. 1999;37:846-857.

24. Charlson ME, Pompei P, Ales KL, MacKenzie CR. A new method of classifying prognostic comorbidity in longitudinal studies: development and validation. J Chronic Dis. 1987;40:373-383.

25. Ashcraft MLF, Fries BE, Nerenz ER, et al. A psychiatric patient classification system. An alternaitve to diagnosisrelated groups. Med Care. 1989;27:543-557.

26. Faries DE, Leon AC, Haro JM, Obenchain RL. Analysis of Observational Healthcare Data Using SAS. Cary, NC: SAS Institute Inc.; 2010.

27. Seeger JD, Williams PL, Walker AM. An application of propensity score matching using claims data. Pharmacoepidemiol Drug Saf. 2005;14:465-476.

28. Diehr P, Yanez D, Ash A, Hornbrook M, Lin DY. Methods for analyzing health care utilization and costs. Annu Rev Public Health. 1999;20:125-144.

Address correspondence to: Shirley Musich, PhD, Senior Research Director Advanced Analytics, Optum 315 E. Eisenhower Parkway, Suite 305 Ann Arbor, MI 48108

E-mail: shirley.musich@optum.com 
This article has been cited by:

1. Rachel Hale, Jeannie Giese. 2017. Cost-Effectiveness of Health Coaching. Professional Case Management 22:5, 228-238. [Crossref]

2. Gibril J. Njie, Ramona K.C. Finnie, Sushama D. Acharya, Verughese Jacob, Krista K. Proia, David P. Hopkins, Nicolaas P. Pronk, Ron Z. Goetzel, Thomas E. Kottke, Kimberly J. Rask, Daniel T. Lackland, Lynne T. Braun. 2015. Reducing Medication Costs to Prevent Cardiovascular Disease: A Community Guide Systematic Review. Preventing Chronic Disease 12. . [Crossref] 